\title{
Tp53 Mutation Inhibits Ubiquitination and Degradation of WISP1 via Down-Regulation of Siah1 in Pancreatic Carcinogenesis
}

OPEN ACCESS

Edited by:

Ruixin Zhu,

Tongji University, China

Reviewed by:

Shasha Song,

Dalian Medical University, China

Chaoyang Li,

Wuhan Institute Of Virology (CAS),

China

Assia Angelova,

Deutsches Krebsforschungszentrum, Helmholtz-Gemeinschaft Deutscher

Forschungszentren (HZ), Germany

${ }^{*}$ Correspondence:

Lifu Wang

lifuwang@sjtu.edu.cn

Qi Wang

wangqilaile@hotmail.com

Ying Zhu

zhuying@medmail.com.cn

${ }^{\dagger}$ These authors have contributed equally to this work.

Specialty section: This article was submitted to Gastrointestinal and Hepatic Pharmacology,

a section of the journal Frontiers in Pharmacology

Received: 26 April 2018

Accepted: 16 July 2018

Published: 03 August 2018

Citation:

Wu W, Liu X, Wei L, Li T, Zang Y, Qian Y, Bai T, Li J, Xie M, Zhu Y, Wang $Q$ and Wang $L$ (2018) Tp53 Mutation Inhibits Ubiquitination and Degradation of WISP1 via

Down-Regulation of Siah1

in Pancreatic Carcinogenesis.

Front. Pharmacol. 9:857.

doi: 10.3389/fphar.2018.00857
Wei Wut, Xu Liut, Lumin Weit, Tong Lit, Yi Zang, Yuting Qian, Tingting Bai, Juanjuan Li, Mingping Xie, Ying Zhu*, Qi Wang* and Lifu Wang*

Department of Gastroenterology, Ruijin Hospital Affiliated to The Shanghai Jiao Tong University School of Medicine, Shanghai, China

Wnt1 inducible signaling pathway protein-1 (WISP1) may play an important role in promoting carcinogenesis. However, the biological function and underlying mechanism of WISP1 in pancreatic carcinogenesis still remains enigmatic. In this study, immunochemistry staining showed that protein levels of WISP1 were more significantly upregulated in pancreatic ductal adenocarcinoma (PDAC) tissues with Tp53 mutation than in PDAC tissues with Tp53 wild-type. In addition, a significant correlation was observed between increased malignant phenotype of tumors from well-differentiated adenocarcinoma tissues to moderately- or poorly-differentiated adenocarcinoma tissues shifting from cytoplasmic expression to nuclear accumulation of WISP1. Interestingly, WISP1 expression was correlated with the poor prognosis in PDAC patients with Tp53 mutation. Also, the biological function analysis showed that WISP1 may act as a potential oncogene in PDAC cells. In addition, immunofluorescence analysis showed that Tp53 mutation promoted WISP1 expression in PanIN and PDAC cells, while Siah E3 Ubiquitin Protein Ligase 1 (Siah1) inhibited WISP1 expression in PDAC cells. Moreover, through immunoprecipitation, immunoblotting analysis, in vitro binding assay, and ubiquitination assay, we found that Tp53 mutation inhibited ubiquitination and degradation of Siah1-dependent WISP1. Therefore, Tp53 mutationSiah1-WISP1 is a new signaling pathway, playing an important role in pancreatic carcinogenesis.

Keywords: WISP1, Tp53 mutation, Siah1, pancreatic cancer, carcinogenesis

\section{INTRODUCTION}

Pancreatic ductal adenocarcinoma is one of the most malignant tumors of the gastrointestinal tract and its incidence grows with the social and economic development levels. In spite of continuous efforts on its early diagnosis and treatment, in the recent 5 years, the survival rate of pancreatic cancer still remains as low as 9\% (Siegel et al., 2018). The known suppressors Tp53, Smad4, and

Abbreviations: MD-PDAC: moderately-differentiated pancreatic ductal adenocarcinoma; PanIN, pancreatic intraepithelial neoplasia; PDAC, Pancreatic ductal adenocarcinoma; PD-PDAC, poorly-differentiated pancreatic ductal adenocarcinoma; WD-PDAC, well-differentiated pancreatic ductal adenocarcinoma; WISP1, Wnt1 inducible signaling pathway protein-1. 
p16 are frequently inactivated in PDAC. Tp53 mutation is detected in 50-70\% of PDAC patients (Rosenfeldt et al., 2013), disturbing normal cell functions. Wnt signaling pathway is highly conservative and its relevant mutations are universal among PDAC patients (Jones et al., 2008). Our previous study has also showed a correlation between Tp53 mutation and WISP1 (Wang et al., 2015). WISP1 is a matricellular protein and plays a significant role in regulation of cellular signaling networks (Berschneider and Konigshoff, 2011). Recently, abnormal expression of WISP1 has been proven in various types of human malignancies (Gurbuz and Chiquet-Ehrismann, 2015; Chahal et al., 2016; Wu et al., 2016; Jing et al., 2017). A previous study demonstrated that WISP1 protects human lung and breast cancer cells from p53-dependent cell death, suggesting that there could be a crosstalk between Tp53 and WISP1 signaling pathways (Su et al., 2002). Nevertheless, the mechanism behind remains unknown.

Recently, several studies showed that Tp53 may promote Siah1 protein levels, which is an E3 ubiquitin-protein ligase that mediates ubiquitination and subsequent proteasomal degradation of target proteins (Fujita et al., 2010; Yuan et al., 2017). These findings motivated us to examine whether E3 is an ubiquitin ligase SIAH1 mediates ubiquitination and degradation of WISP1. In our study, WISP1 was probably an oncogene, and its protein level was observed more significant for upregulation in PDAC tissues and PDAC cells with Tp53 mutation than in PDAC tissues and PDAC cells with Tp53 wild-type. Moreover, we attempted to demonstrate that Tp53 mutation may downregulate Siah1 protein levels, which may inhibit ubiquitination and degradation of Siah1-dependent WISP1 and induce WISP1 nuclear import.

\section{MATERIALS AND METHODS}

\section{Patients and Tissue Samples}

In this study, 203 PDAC and paraneoplastic tissues post operation were retrospectively obtained from Ruijin Hospital (Shanghai, China) before 2017. The consent of participants was obtained for PDAC research. None of the patients had undergone radiotherapy or chemotherapy before surgery. The tissues were embedded in paraffin wax for analysis. Histological diagnoses were performed by two independent senior pathologists. This study was carried out in accordance with the recommendations of the Ethics Committee of Ruijin Hospital, affiliated with Shanghai Jiao Tong University, School of Medicine with written informed consent from all subjects. All subjects gave written informed consent in accordance with the Declaration of Helsinki. The protocol was approved by the Ethics Committee of Ruijin Hospital, affiliated with Shanghai Jiao Tong University, School of Medicine.

\section{Cell Lines}

Low-passage-number cells (P8) of the preinvasive pancreatic ductal cell line SH-PAN isolated from
Pdx-1-Cre; LSL-Kras ${ }^{G 12 D /+}$ mutant mice was employed. The SH-PAN cell line has only Kras mutation (Hingorani et al., 2003, 2005). Human PDAC cell lines with wild-type Tp53 (Capan-2, HPAC) and Tp53 mutants (Panc-1, MIA PaCa-2, HPAF-II-1, BxPC-3, AsPC-1), were purchased from the American Type Culture Collection (Sipos et al., 2003; Deer et al., 2010). Pancreatic carcinoma cell lines were cultured in Dulbecco's modified Eagle's medium (DMEM) (Panc-1, HPAC, HPAF-II), RPMI-1640 medium (AsPC-1 and BxPC-3), McCoy's 5a medium (MIA PaCa-2), and Iscove's Modified Dulbecco's medium (Capan-2). All cells cultured in the abovementioned media were supplemented with $10 \%$ heat-inactivated fetal bovine serum (FBS) at $37^{\circ} \mathrm{C}$ with $5 \% \mathrm{CO}_{2}$.

\section{Reagents}

MG132 (Proteasome inhibitor), Cycloheximide (inhibitor of protein synthesis in eukaryotes), and Nutlin-3a (inhibitor of the MDM2-p53 interaction) were purchased from Sigma-Aldrich (St. Louis, MO, United States).

\section{Plasmid Constructs and Lentivirus-Mediated shRNA or Gene Overexpression}

The shRNA target sequences containing four different sequences of humanWISP1 (GenBank accession number: NM_003882.3) and human Siah1 (GenBank accession number: NM_003031.3) were selected for shRNA interference. The mouse Tp53 (GenBank accession number: NM_011640.3) cDNA fragment was polymerase chain reaction PCR-amplified and mutated in Tp53 $3^{R 172 H}$. The human Tp53 (GenBank accession number: NM_001276760.1) cDNA fragment was PCR-amplified and mutated in $T p 53^{R 175 H}$ or $T p 53^{R 273 H}$. Lentivirus-mediated overexpression of the mouse $T p 53^{R 172 H}$, the human $T p 53^{R 175 H}$, the human $T p 53^{R 273 H}$, the human wild-type Tp53, and the mouse WISP1 (GenBank accession number: NM_018865.2) was constructed using the pGLV5-EF1a-GFP vectors (GenePharma, Shanghai, China). The full-length human Siah-1 cDNA was cloned into pGEX-KG (American Type Culture Collection, Manassas, VA, United States) to generate E. coli expression constructs of GST-Siah-1 fusion proteins. For synthesis of in vitro protein, the full-length WISP1 cDNA was cloned into pEXP1-DEST vector (Invitrogen, Carlsbad, CA, United States) to produce a polyhistidine tagged WISP1 (His6-WISP1) and a polypeptide protein tagged WISP1(Myc-WISP1). The cDNA fragment for GST-Siah-1 fusion protein (fulllength, wild-type Siah-1; as in pGEX-KG) was also cloned in pEXP1-DEST. pRK5-HA-Ub for overexpression of HAtagged ubiquitin was previously described (Conze et al., 2008). All constructs were verified by DNA sequencing. The lentivirus vector transduction was performed as previously described (Wang et al., 2016). Transient transfection of plasmid DNA was performed using Lipofectamine 2000 transfection reagent (Invitrogen). Cells were grown in culture medium with $10 \mu \mathrm{g} / \mathrm{ml}$ puromycin for selection of stable cells (Wang et al., 2013, 2016). Primers used are shown in Table 1. 
TABLE 1 | Primers used in this study.

\begin{tabular}{|c|c|}
\hline Name & \\
\hline \multicolumn{2}{|c|}{ Siah1 shRNA (human) } \\
\hline SR-1F & 5'-GATCCGTCTTAGAGAAACAGGAAATTCAAGAGATाTCCTGTाTCTCTAAGAC $\Pi 1 \Pi \Pi \mathrm{G}-3^{\prime}$ \\
\hline SR-1R & 5'-AATTCAAAAAAGTCTTAGAGAAACAGGAAATCTCTTGAATTCCTGTTCTCTAAGACG-3' \\
\hline SR-2F & 5'-GATCCAAGGAATTGCAACAGCCATTCAAGAGATGGCTGTTGCAATTCCTा ПाПTG-3' \\
\hline SR-2R & 5'-AATTCAAAAAA AAGGAATTGCAACAGCCATCTCTTGAATGGCTGTTGCAATTCCTT G-3' \\
\hline SR-3F & 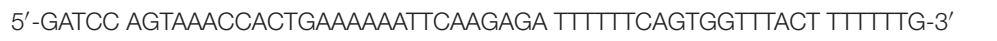 \\
\hline SR-3R & 5'-AATTCAAAAAA AGTAAACCACTGAAAAAATCTCTTGAA ПIIITCAGTGGTITACT G-3' \\
\hline $\mathrm{SR}-4 \mathrm{~F}$ & 5'-GATCC AGTATC ПITGATATGCTTCAAGAGA GCATATCAAAAGATAC $\Pi 11 \Pi \Pi G$-3' \\
\hline SR-4R & 5'-AATTCAAAAAA AGTATCTITGATATGCTCTCTTGAA GCATATCAAAAGATACT G-3' \\
\hline \multicolumn{2}{|c|}{ WISP1 shRNA (human) } \\
\hline WR-1F & 5'-GATCC TAGGAGTGTGTGCACAGGTTCAAGAGA CCTGTGCACACACTCCTA Tा11TG-3' \\
\hline WR-1R & 5'-AATTCAAAAAA TAGGAGTGTGTGCACAGGTCTCTTGAA CCTGTGCACACACTCCTA G-3' \\
\hline WR-2F & 5'-GATCC GCACACGCTCCTATCAACTTCAAGAGA GTTGATAGGAGCGTGTGC TाIITG-3' \\
\hline WR-2R & 5'-AATTCAAAAAA GCACACGCTCCTATCAACTCTCTTGAA GTTGATAGGAGCGTGTGC G-3' \\
\hline WR-3F & 5'-GATCC GAAATGGAAT CAGGTAGATTCAAGAGA TCTACCTGATTCCATTC $\Pi 11 T G-3^{\prime}$ \\
\hline WR-3R & 5'-AATTCAAAAAA GAAATGGAAT CAGGTAGATCTCTTGAA TCTACCTGATTCCATITC G-3’ \\
\hline WR-4F & 5'-GATCC CTCTTATAGT CTICTAGTTCAAGAGA CTAGAAAGACTATAAGAG ПIIITG-3' \\
\hline WR-4R & 5'-AATTCAAAAAA CTCTTATAGT CTITCTAGTCTCTTGAA CTAGAAAGACTATAAGAG G-3' \\
\hline $\mathrm{NC}-\mathrm{F}$ & 5'-GATCCTTGCGCAACTGTGTCACGTTCAAGAGAACGTGACACAGTTGCGCAATIIITG-3' \\
\hline NC-R & 5'-AATTCAAAAAATTGCGCAACTGTGTCACGTTCTCTTGAAACGTGACACAGTTGCGCAAG-3' \\
\hline
\end{tabular}

\section{Immunoprecipitation and Immunoblotting}

Cells were lysed by sonicating for $5 \mathrm{~s}$ in $1 \mathrm{ml}$ of detergentfree lysis buffer [phosphate buffered saline (PBS), $5 \mathrm{mM}$ of Ethylenediaminetetraacetic acid (EDTA], 0.02\% sodium azide, $10 \mathrm{mM}$ of iodoacetamide, $1 \mathrm{mM}$ of phenylmethane sulfonyl fluoride (PMSF), and $2 \mathrm{mg}$ of leupeptin) at $4^{\circ} \mathrm{C}$. Antibodyconjugated beads were prepared by combining $1 \mathrm{mg}$ of antibodies with $30 \mathrm{ml}$ of a $50 \%$ protein. A Sepharose bead slurry in $0.5 \mathrm{ml}$ of ice-cold PBS was incubated for $1 \mathrm{~h}$ at $4^{\circ} \mathrm{C}$ in a tube rotator and were then washed three times with $1 \mathrm{ml}$ of lysis buffer. The antibodies used for coimmunoprecipitation were Akt (ab6076, Abcam, Cambridge, United Kingdom). The beads were washed three times with washing buffer $(50 \mathrm{mM}$ of Tris- $\mathrm{HCl}$, $300 \mathrm{mM}$ of $\mathrm{NaCl}, 5 \mathrm{mM}$ of EDTA, $0.02 \%$ sodium azide, and $0.1 \%$ Triton X-100) and once with ice-cold PBS. Immunodetection was carried out using the ECL Western Blotting Detection Kit (Amersham Corp., Burlington, MA, United States). Proteins were detected using antibodies against WISP1 (ab178547, Abcam, Cambridge, United Kingdom), wild-type Tp53 (ab31333, Abcam, Cambridge, United Kingdom), Siah1 (ab2237, Abcam, Cambridge, United Kingdom), Myc tag (ab9106, Abcam, Cambridge, United Kingdom), HA tag (ab9110, Abcam, Cambridge, United Kingdom), and anti-GST (ab19256, Abcam, Cambridge, United Kingdom); and glyceraldehyde 3-phosphate dehydrogenase (GAPDH) levels were used as an internal reference control for the relative protein expression levels.

\section{In Vitro Binding Assay}

In vitro protein synthesis of His6-tagged WISP1, GST and GST-Siah-1 fusion protein was performed using Expressway cell-free E. coli expression system (Invitrogen, Waltham, CA,
United States), followed by purification of His6-tagged WISP1 using Ni-NTA magnetic agarose beads (Qiagen, München, Germany) and of GST and GST-Siah-1 using glutathioneSepharose 4FF (GE Healthcare, Piscataway, NJ, United States). The eluted proteins were analyzed by immunoblot as previously described.

\section{Ubiquitination Assay}

The cells were transfected with Siah-1 shRNA or control shRNA for $24 \mathrm{~h}$, and then transfected with pEXP1-DEST-MycWISP1, pRK5-HA-Ub, and pGLV5-p53 plasmids. After $24 \mathrm{~h}$, the cells were treated with $15 \mu \mathrm{M}$ of MG132 for $8 \mathrm{~h}$ and lysed in the buffer containing $50 \mathrm{mM}$ of 4-(2-hydroxyethyl)-1piperazineethanesulfonic acid (HEPES, pH 7.5), $150 \mathrm{mM}$ of NaCl, $1 \%$ Triton X-100, $1 \%$ glycerol, $10 \mu \mathrm{M}$ of MG132, $100 \mathrm{mM}$ of NaF, $1 \mathrm{mM}$ of NaVO3, $25 \mathrm{mM}$ of $\beta$-glycerophosphate, and complete protease inhibitors. Immunoprecipitation and immunoblot were performed as described above.

\section{Cell Proliferation, Soft Agar Assay, and Invasion Assay}

For the cell proliferation assay, cells were seeded in a 96-well plate at a concentration of $5 \times 103$ cells/well 1 day before the experiment. 3-[4,5-Dimethylthiazol-2-yl]-2,5diphenyltetrazolium bromide (MTT, $0.5 \mathrm{mg} / \mathrm{ml}$, Sigma, St. Louis, MO, United States) was added to each well 1, 2, 3, 4, and 5 days after seeding. Cells were cultured at $37^{\circ} \mathrm{C}$ for $4 \mathrm{~h}$, followed by addition of $150 \mu \mathrm{l}$ of dimethylsulfoxide (DMSO). Absorption was measured at a wave length of $490 \mathrm{~nm}$. For the soft agar assay, cells were seeded in six-well plates for the soft agar assay. Each well contained a bottom layer of $1 \%$ agarose, a middle layer of $0.5 \%$ agarose that included 1000 cells, and a top layer 
of medium, which was changed every 6 days. After 26 days, colonies were counted by Quantityone analysis software (BioRad Inc., Hercules, CA, United States). The transwell invasion assay was performed using a Millicell invasion chamber (Millipore, Burlington, MA, United States). The $8-\mu \mathrm{m}$ pore inserts were coated with $15 \mu \mathrm{g}$ of Matrigel (Becton Dickinson Labware, Bedford, MA, United States), and $5 \times 10^{4}$ cells were seeded in the top chamber. The Matrigel invasion chamber was incubated for $20 \mathrm{~h}$ in a humidified tissue culture incubator. Non-invading cells were removed from the top of the Matrigel with a cottontipped swab. Invading cells on the bottom surface of the filter were fixed in methanol and stained with crystal violet. Invasion ability was determined by counting the stained cells (Wang et al., 2013, 2016).

\section{Nude Mouse Subcutaneous Xenograft and Metastasis Model}

The BALB/c nude mice were divided into experimental group and control group. The cells $\left(5 \times 10^{6}\right.$ per mouse $)$ were injected into the subcutis of nude mice through five of experimental or control group, and 25 days later, tumor volume $(\mathrm{V})$ was estimated using the formula $\mathrm{V}=\mathrm{LW}^{2} / 6$ ( $\mathrm{L}$ : length of tumor; $\mathrm{W}$ : width of tumor). Also, the cells $\left(1 \times 10^{6}\right.$ per mouse $)$ were injected into the tail vein of nude mice in groups of five of experimental or control group, and 60 days later each mouse's lung was removed and lung metastases were determined. This study was carried out in accordance with the recommendations of the Institutional Animal Care and Use Committee of the Shanghai Experimental Animals Centre of Chinese Academy of Sciences. The protocol was approved by the Institutional Animal Care and Use Committee of the Shanghai Experimental Animals Centre of Chinese Academy of Sciences.

\section{Histology, Immunohistochemistry, and Immunofluorescence}

Four-micrometer-thick sections were stained with hematoxylin and eosin for histological verification. Primary antibodies against WISP1 (ab178547, Abcam, Cambridge, United Kingdom) and Siah1 (ab2237, Abcam, Cambridge, United Kingdom) were purchased. To evaluate the immunohistochemistry results, the percentage of positive cells was scored from grade 0 to grade 3: Grade 0 (negative), $<1 \%$ or $0 \%$ of the cells were stained; Grade 1 (weak), $1-49 \%$ of the cells were stained; Grade 2 (moderate), $50-70 \%$ of the cells were stained; and Grade 3 (intense), $>70 \%$ of the cells were stained (Hotz et al., 2007; Shen et al., 2013; Wang et al., 2016). For immunofluorescence staining, the antibodies used included WISP1 (ab178547, Abcam, Cambridge, United Kingdom), Tp53 (ab16465, Abcam, Cambridge, United Kingdom) and Siah1 (ab2237, Abcam, Cambridge, United Kingdom). Cells were then fixed with $4 \%$ Paraformaldehyde and permeabilized with $0.1 \%$ Triton $\mathrm{X}-100$. The antibodies were applied at $4^{\circ} \mathrm{C}$ overnight. FITC-conjugated goat anti-mouse IgG, Rhodamine-conjugated goat anti-rabbit IgG and FITC-conjugated rabbit anti-goat IgG were used as secondary antibodies. Fluorescently-labeled secondary antibodies were added and incubated for $1 \mathrm{~h}$ at room temperature. Eventually, nuclei were counterstained with DAPI. The stained cells were mounted on glass slides and examined by confocal microscopy (Wang et al., 2016; Li et al., 2017).

\section{Statistical Analysis}

Statistical analyses were carried out using SPSS 19.0 software (SPSS Inc., Chicago, IL, United States). Each experiment was repeated at least three times. The results were presented as the mean \pm standard deviation (SD). Student's $t$-test and KruskalWallis test were used to assess the statistical significance of differences among the different groups. Postoperative survival was evaluated using the Kaplan-Meier method and log-rank test. Pearson's correlation test was used for correlation analysis. $P$-value less than 0.05 was statistically considered significant.

\section{RESULTS}

\section{The Expression and Clinical Significance of WISP1 in PDAC Tissues With Tp53 Mutation}

To determine whether the differential expression of WISP1 was correlated with $T p 53$ mutation, immunohistochemistry staining was performed to assess the WISP1 levels in 203 PDAC tissues (including 82 tissues with wild-type $T p 53$ and 121 ones with $T p 53$ mutation) and 203 paraneoplastic tissues. Samples were scored from grade 0 to 3 as previously described in MATERIALS AND METHODS. For 203 paraneoplastic tissue samples, negative, weak, moderate, or intense WISP1 staining was observed in 87 , 80,31 , and 5 tissues, respectively. For 82 PDAC tissues with wildtype Tp53, negative, weak, moderate, or intense WISP1 staining was observed in $27,31,19$, and 5 tissues, respectively.

In contrast, negative, weak, moderate, or intense WISP1 staining was observed in $4,13,16$, and 15 of the 48 welldifferentiated adenocarcinoma tissues (WD-PDAC) with Tp53 mutation and in 5, 12, 25, and 31 of the 73 moderatelyor poorly-differentiated adenocarcinoma tissues (MD- or PDPDAC) with Tp53 mutation (Figures 1A,B). In addition, negative or weak WISP1 cytoplasmic staining, moderate or strong WISP1 cytoplasmic staining, and WISP1 nuclear staining were observed in 11,29, and 8 of the 48 WD-PDAC tissues, and 13, 16, and 44 of the $73 \mathrm{MD}$ or PD-PDAC tissues (Figure 1C). These data showed that WISP1 protein levels were significant upregulated in PDAC tissues with Tp53 mutation than in PDAC tissues with wild-type $T p 53$. In addition, a significant correlation was observed between increased malignant phenotype of tumors from WD-PDAC to MD- or PD-PDAC and shift from cytoplasmic expression to nuclear accumulation of WISP1.

Moreover, the PDAC samples with wild-type Tp53 or Tp53 mutation were divided into two groups for clinicopathological evaluation according to the WISP1 levels respectively: moderate or strong WISP1 staining (Grade 2 or 3 ), and negative or weak WISP1 staining (Grade 0 or 1). Kaplan-Meier analysis showed that the postoperative median survival was 11.78 months for patients with moderate or strong WISP1 staining and 14.51 months for patients with negative or weak WISP1 staining, 
A

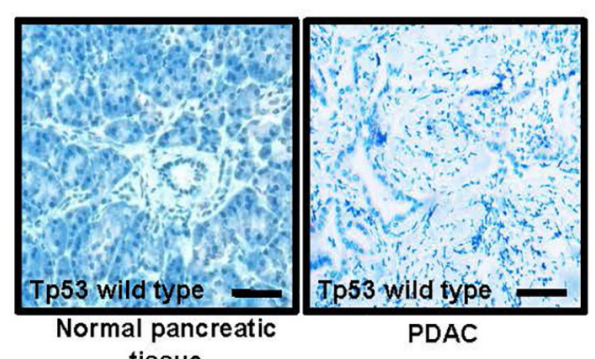

tissue

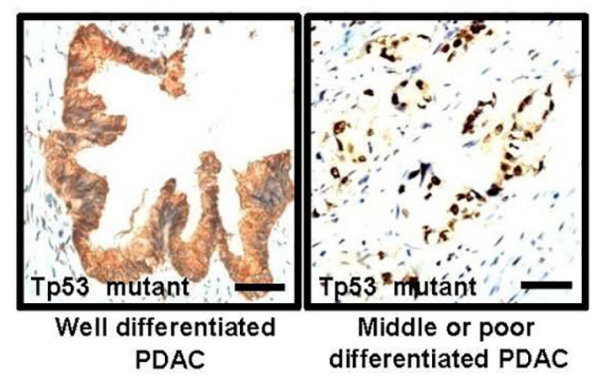

B

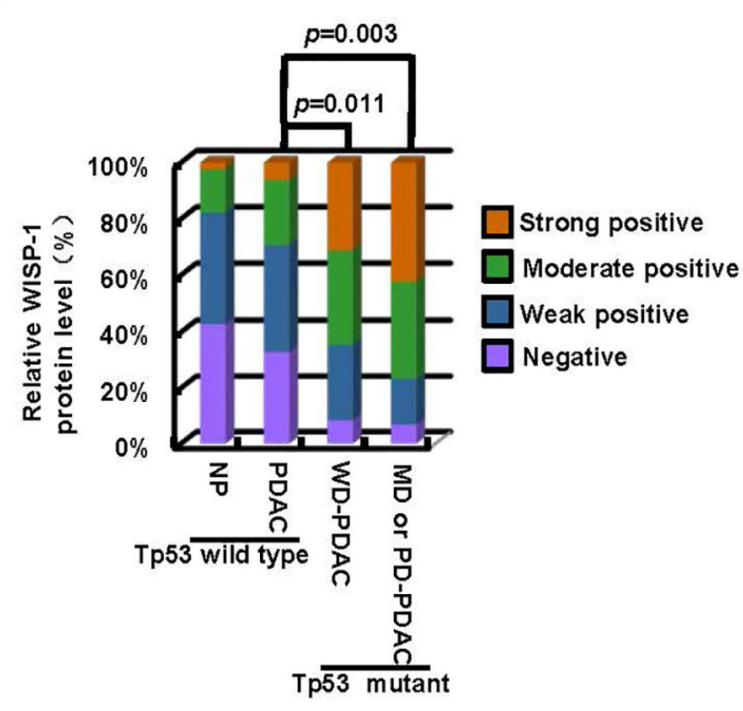

C

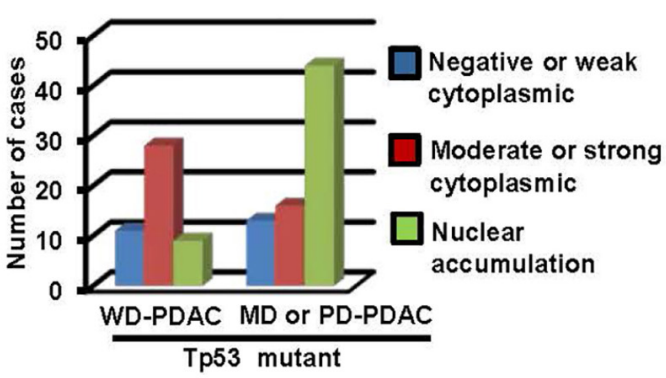

D

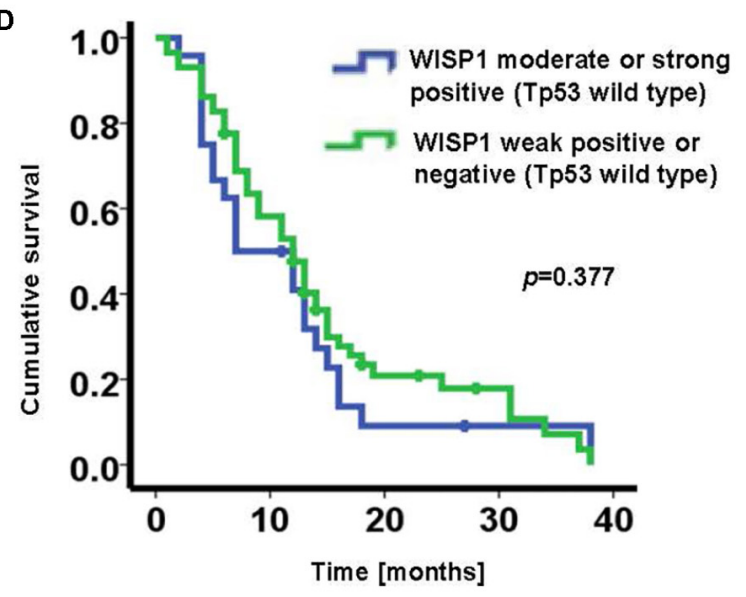

E

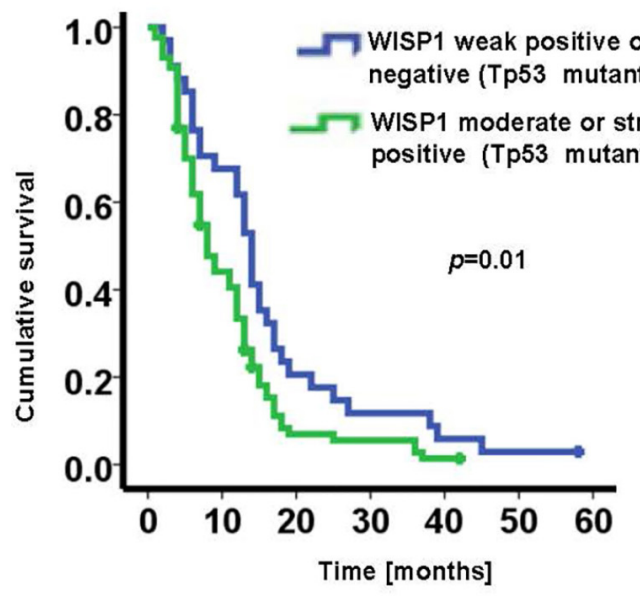

$\mathbf{F}$

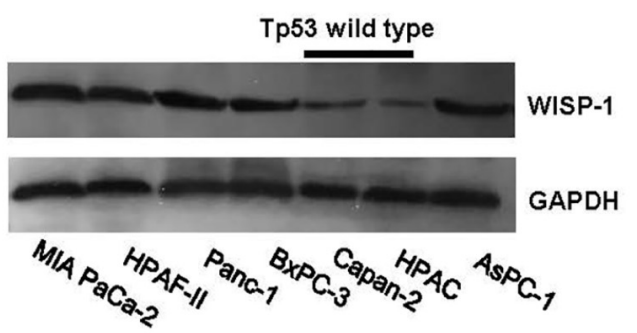

FIGURE 1 | The expression and clinical significance of WISP1 in PDAC with Tp53 mutation. (A-C) Immunohistochemistry staining of WISP1 was summarized in the histogram. WD-PDAC, MD-PDAC, and PD-PDAC represent well, moderately, and poorly-differentiated adenocarcinoma, respectively (black bar scale = $100 \mu \mathrm{m})$. (D) Kaplan-Meier survival curves for moderate or strong WISP1 staining (Grade 2 or 3) and negative or weak WISP1 staining (Grade 0 or 1) in patients with wild-type Tp53 were performed using the log-rank test. (E) Kaplan-Meier survival curves for moderate or strong WISP1 staining (Grade 2 or 3) and negative or weak WISP1 staining (Grade 0 or 1 ) in patients with Tp53 mutation were performed using the log-rank test. (F) WISP1 was detected in PDAC cell lines with or without Tp53 mutation. 
as illustrated in Figure 1D (wild-type Tp53) $(P=0.377)$. In contrast, the postoperative median survival was 10.70 months for patients with moderate or strong WISP1 staining and 16.12 months for patients with negative or weak WISP1 staining, as showed in Figure 1E (Tp53 mutation) $(P=0.01)$. These data demonstrated that WISP1 upregulation may contribute to the poor prognosis in PDAC patients with Tp53 mutation.

Furthermore, we investigated whether WISP1 expression was associated with Tp53 mutation in human PDAC cell lines. The WISP1 protein levels in MIA PaCa-2, HPAF-II and Panc-1, BxPC-3 and AsPC- 1 cells with Tp53 mutation were significantly higher than those in Capan-2 and HPDC cells with wild-type Tp53 (Figure 1F) $(n=3)$.

\section{WISP1 Expression Promoted the Malignancy of Cells}

As the function of WISP1 in PDAC is unclear, we attempted to determine its biological role. Firstly, lentivirus-mediated mouse WISP1-overexpression vector or control was stably transduced into SH-PAN cells (SH-PAN-lent-WISP1 and SH-PAN-lentctr) and lentivirus-mediated human WISP1-silencing vector or control was stably transduced into Panc-1 cells (Panc-1lent-WISP1i and Panc-1-lent-ctr) and MIA PaCa-2 cells (MIA PaCa-2-lent-WISP1i and MIA PaCa-2-lent-ctr). The efficiency of WISP1 overexpression in SH-PAN cell line and WISP1 knockdown in Panc-1 or MIA PaCa- 2 cell line was confirmed by real-time PCR and Western blot analysis (Supplementary Figure S1). MTT assay showed that WISP1 overexpression promoted the growth rate of SH-PAN, and WISP1 silencing attenuated growth rate of Panc- 1 cells and MIA PaCa- 2 cells (Figure 2A) $(n=3)$. Soft agar assay showed that the SH-PANlent-ctr cells did not form colonies while the SH-PAN-lentWISP1 formed $98.7 \pm 14.7$ colonies $(P=0.001)$, and the Panc1-lent-ctr and MIA PaCa-2-lent-ctr cells formed $416.3 \pm 37.2$ and $378 \pm 28.6$ colonies respectively $(P=0.001)$, while the Panc-1-lent-WISP1i and MIA PaCa-2-lent-WISP1i cells only formed $89 \pm 9.4(P=0.001)$ and $76 \pm 8.8(P=0.001)$ colonies respectively, after 26 days (Figure $2 \mathrm{~B})(n=3)$. The invasion assay showed that the overexpression of WISP1 strengthened the invasive ability of SH-PAN (6.3 \pm 2 vs. $31.7 \pm 5.2$ of stained cells, $P=0.003)$, and WIPS1 silencing attenuated the invasive ability of Panc-1 (43 \pm 4.9 vs. $14.3 \pm 2.9$ of stained cells, $P=0.009)$ and MIA PaCa-2 cells (34.3 \pm 3.4 vs. $14.7 \pm 3.1$ of stained cells, $P=0.017$ ) (Figure 2C) $(n=3)$. To determine whether WISP1 plays an important role in the in vivo tumorigenicity and micrometastasis of PDAC cells, Panc-1-lent-WISP1i, MIA PaCa-2-lent-WISP1i cells or the control were injected into the subcutaneous tissue or the tail vein of five nude mice each. The results demonstrated that silencing of WISP1 resulted in a significant decrease in tumorigenicity $(P=0.001)$ (Figure 2D). Moreover, quantification of micrometastasis in lung tissues and H\&E staining showed that WISP1 silencing inhibited the in vivo metastasis of PDAC cells $(10.3 \pm 3.21$ vs. $1.3 \pm 0.58, P=0.007)$ (Figure $2 \mathrm{E}$ ). These data suggest that WISP1 acted as a potential oncogene in PDAC cells.

\section{Tp53 Mutation May Promote WISP1 Expression in SH-PAN and PDAC Cells}

To determine whether WISP1 expression was affected by Tp53, lentivirus-mediated mouse $T p 53^{R 172 H}$ overexpression vector or control was stably transduced into SH-PAN cells, lentivirusmediated human $T p 53^{R 175 H}$ or $T p 53^{R 273 H}$ overexpression vector or control was stably transduced into Capan-2 cells (wild-type Tp53), lentivirus-mediated human wild-type $T p 53$ overexpression vector or control was stably transduced into Panc-1 cells (Tp53 mutation), and then immunofluorescence staining was performed to measure the levels and locations of WISP1 in SH-PAN, Capan-2, and Panc-1 cells. The results showed that nuclear and cytoplasm WISP1 staining was sharply enhanced with overexpression of $T p 53^{R 172 H}$ in SH-PAN cells (Figures 3A,B) and with overexpression of $T p 53^{R 175 H}$ or $T p 53^{R 273 H}$ in Capan-2 cells (Figures 3C,D) $(n=3)$. In addition, the cytoplasm WISP1 staining was sharply enhanced with overexpression of wild-type Tp53 in Panc-1 cells, however, the nuclear WISP1 staining was not affected (Figures 3E,F) $(n=3)$. These data suggest that Tp53 mutation may promote WISP1 expression in SH-PAN cells and PDAC cells.

\section{Siah1 May Inhibit WISP1 Expression in PDAC Cells}

As previous study showed that wild-type Tp53 may promote Siah1 expression which may mediate ubiquitination degradation of downstream target gene, we attempted to investigate whether WISP1 expression was correlated with Siah1.

Lentivirus-mediated Siah1 silencing vector or control was stably transduced into Capan-2 cells (wild-type Tp53), lentivirus-mediated Siah1 overexpression vector or control was stably transduced into Panc-1 cells (Tp53 mutation), and immunofluorescence staining was performed to measure the levels and locations of Siah1 and WISP1 in Capan-2 and Panc-1 cells. The results showed that Siah1 staining was mainly focused on the cytoplasm in PDAC cells, and nuclear and cytoplasm WISP1 staining was sharply enhanced with silencing of Siah1 in Capan-2 cells (Figures 4A,B) $(n=3)$, however, nuclear and cytoplasm WISP1 staining was sharply downregulated with overexpression of Siah1 in Panc-1 cells (Figures 4C,D) $(n=3)$. Moreover, to testify whether Siah1 showed a negative correlation with WISP1 in 203 PDAC tissue samples, the Pearson's correlation analysis was conducted. The results showed that Siah1 expression was significantly and negatively correlated with WISP1 expression in 203 PDAC tissue samples (Figures 4E,F).

The above-mentioned data suggest that Siah1 may inhibit WISP1 expression in PDAC cells.

\section{Tp53 Mutation May Inhibit Siah1-Mediated WISP1 Ubiquitination Degradation}

To further determine whether $T p 53$ mutation may affect the expression of Siah1 or WISP1, lentivirus-mediated 


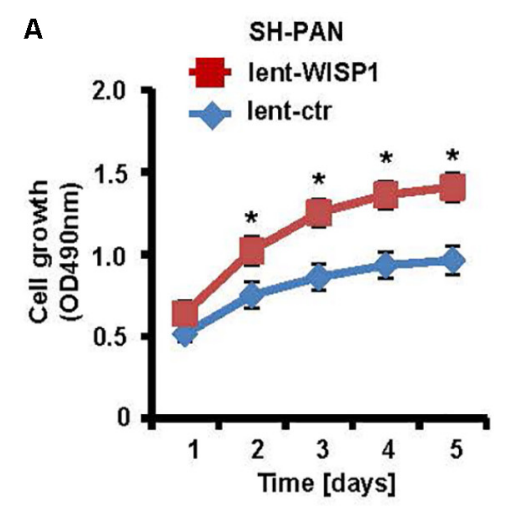

B
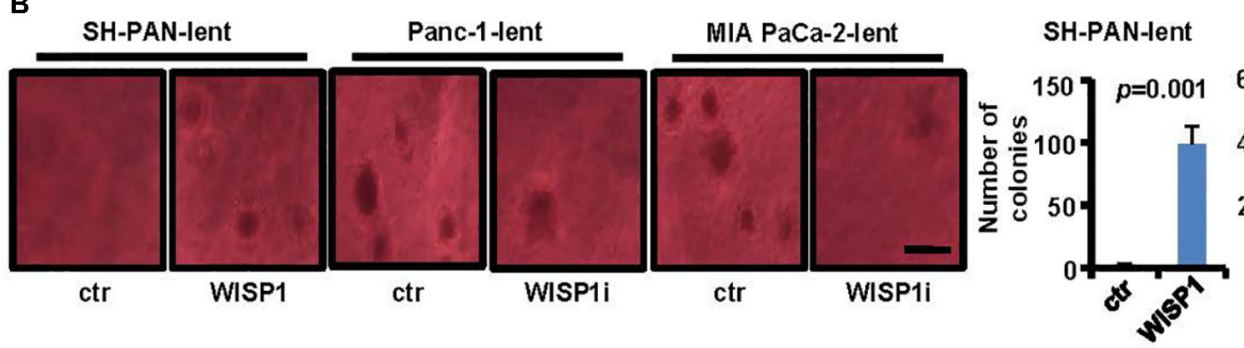

Panc-1-lent MIA PaCa-2-lent

$600{ }_{p=0.001} 4507 \tau^{p=0.001}$

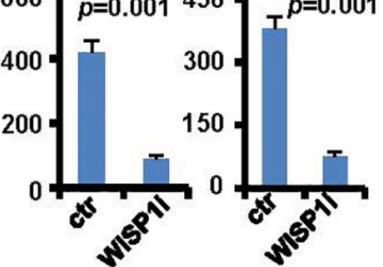

C
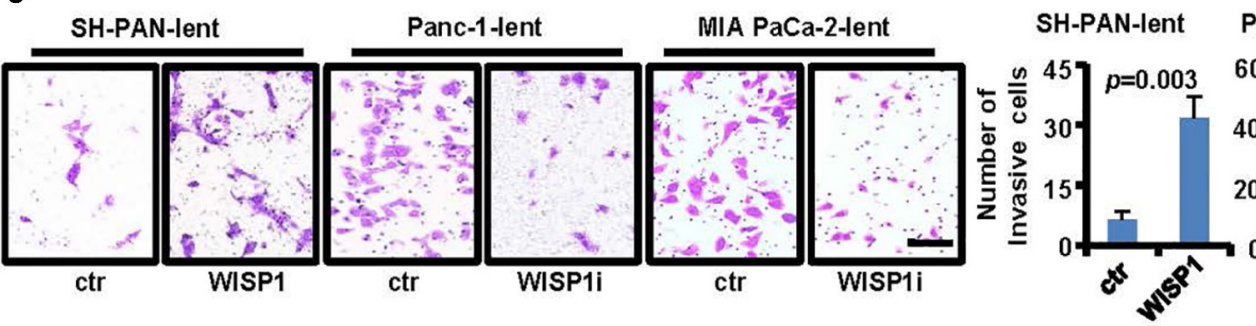

Panc-1-lent MIA PaCa-2-lent

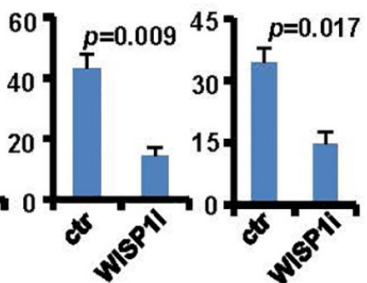

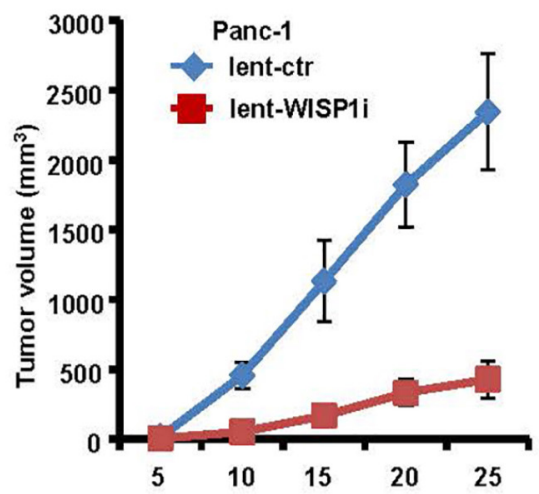

E
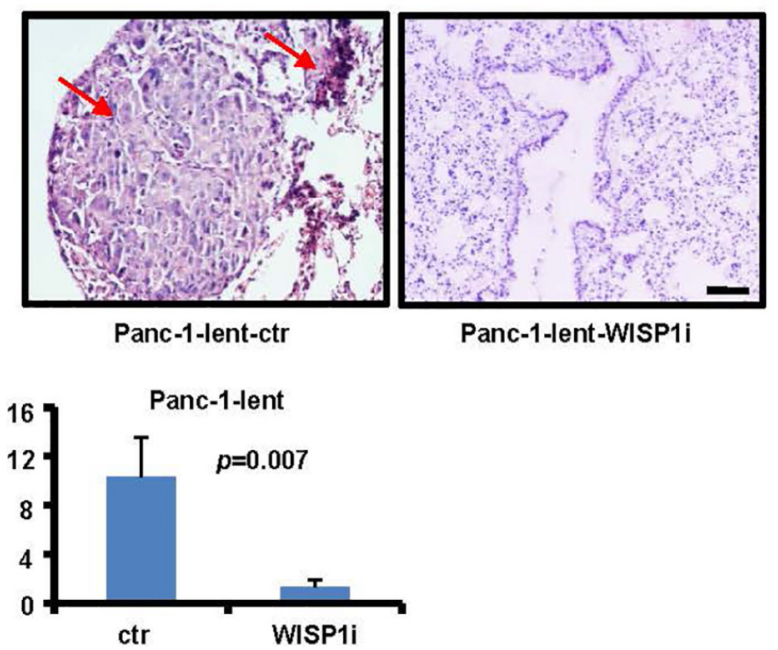

FIGURE 2 | Biological role of WISP1 in pancreatic carcinogenesis. After lentivirus-mediated WISP1-overexpression vector or control (lent-WISP1 or lent-ctr) was transduced into SH-PAN cells and lentivirus-mediated WISP1-silencing vector or control (lent-WISP1 or lent-ctr) was transduced into PDAC cells, MTT assay (A), soft agar assay (B) (black bar scale $=500 \mu \mathrm{m}$ ) and Matrigel invasion assay (C) (black bar scale $=50 \mu \mathrm{m}$ ) showed the anchorage-dependent and anchorage-independent growth ability, and tumor invasion rate. The subcutaneous tumorigenic ability (D) and lung metastasis after vein injection (E) of tumor cells were measured $(n=5)$. HE-stained sections of lung: red arrow, metastatic nodule. ${ }^{*} P<0.05 ;{ }^{* *} P<0.01 ;{ }^{* * *} P<0.001$ (black bar scale $\left.=200 \mu \mathrm{m}\right)$. 


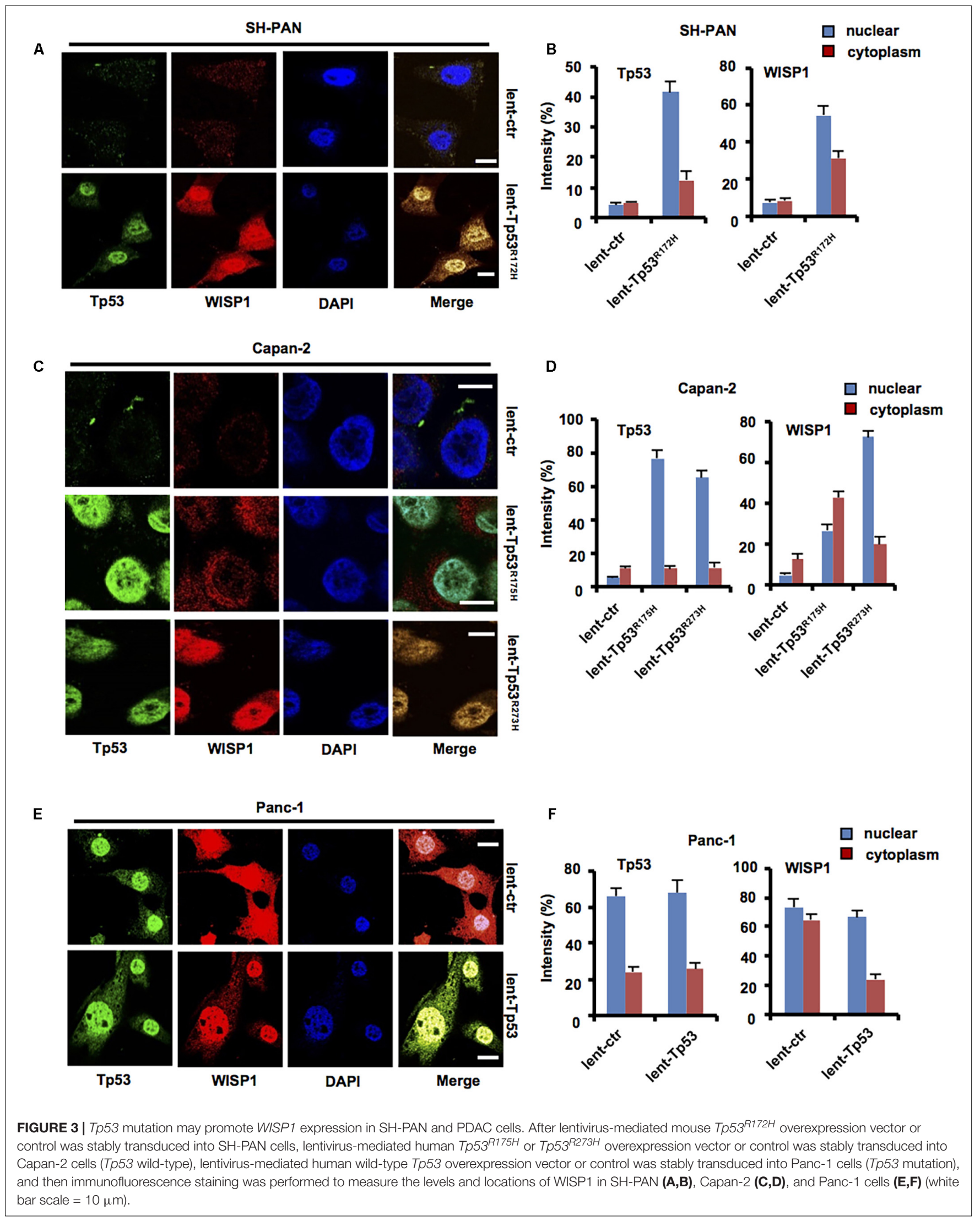




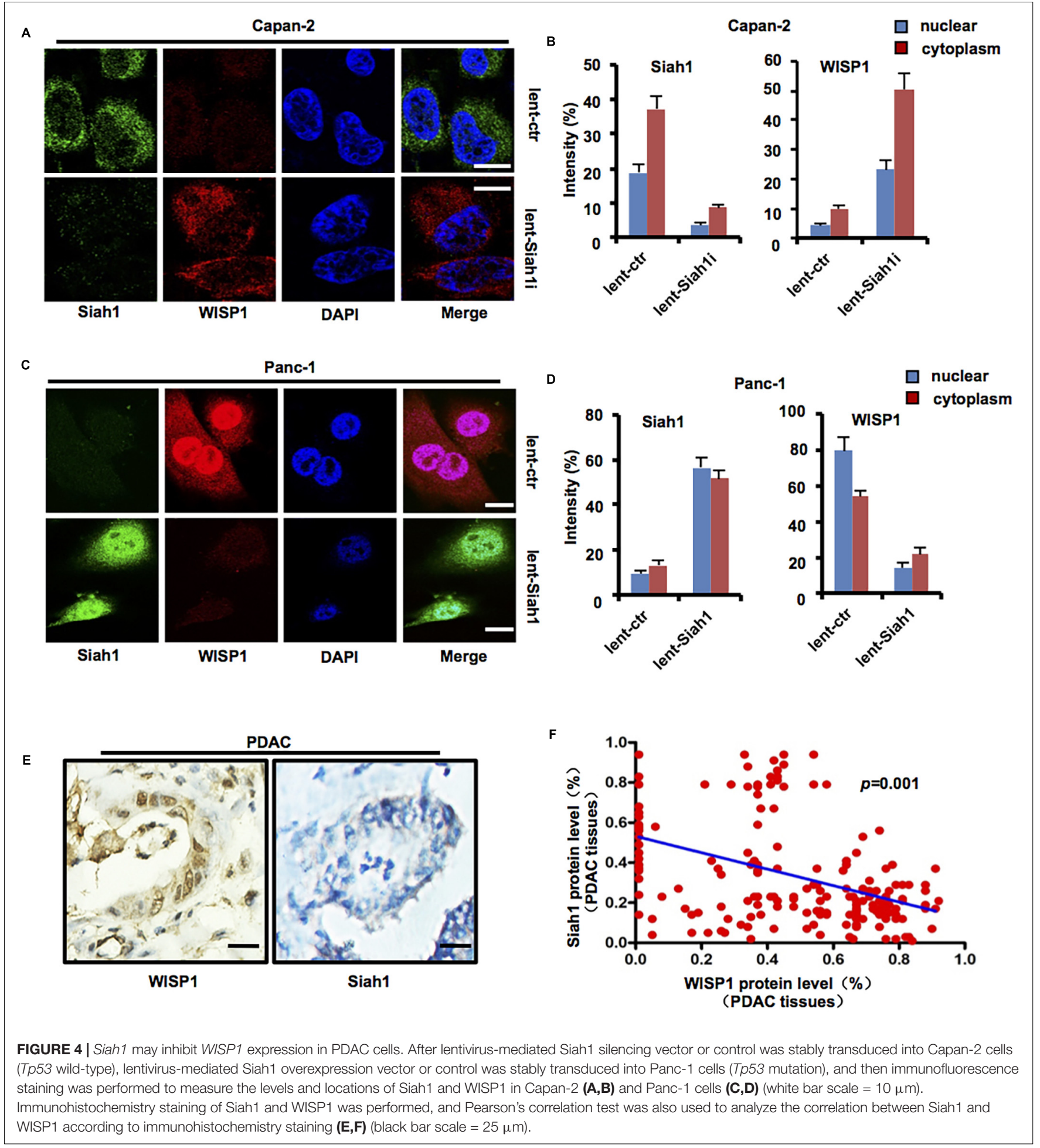

mouse $T p 53^{R 172 H}$ overexpression vector or control was stably transduced into SH-PAN cells, and lentivirusmediated human $T p 53^{R 175 H}$ or $T p 53^{R 273 H}$ overexpression vector or control was stably transduced into Capan-2 cells. Western blot analysis showed that Tp53 mutation downregulated the Siah1 level and upregulated WISP1 level in SH-PAN cells and Capan-2 cells (Figure 5A) $(n=3)$. Also, the stabilization and activation of endogenous Tp53 by the small-molecule inhibitor of MDM2 (Nutlin-3a) $(10 \mu \mathrm{M})$ resulted in an increase in Siah1 and a decrease in WISP1 expression (Figure 5B) $(n=3)$. Moreover, the inhibition of protein synthesis by cycloheximide, followed 
A

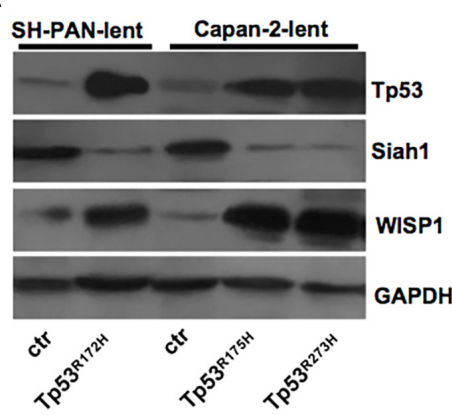

B

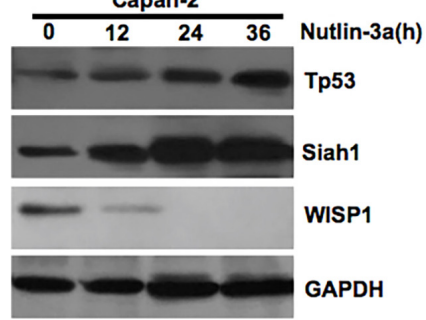

C

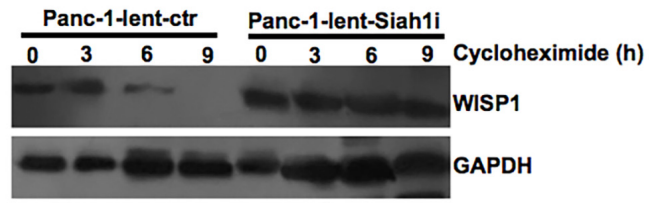

D

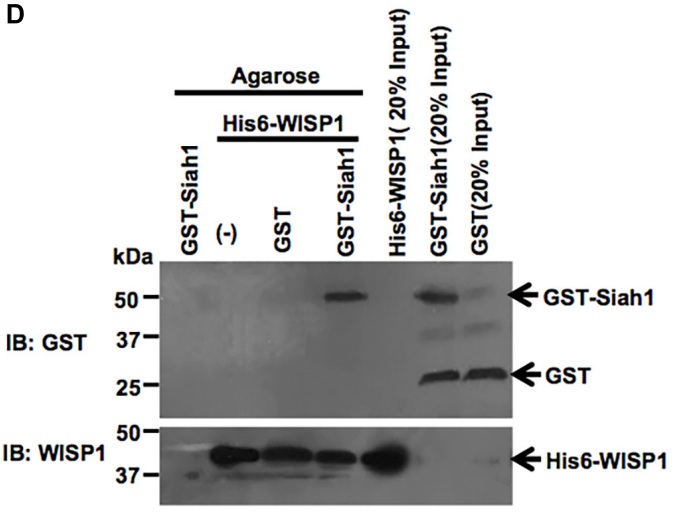

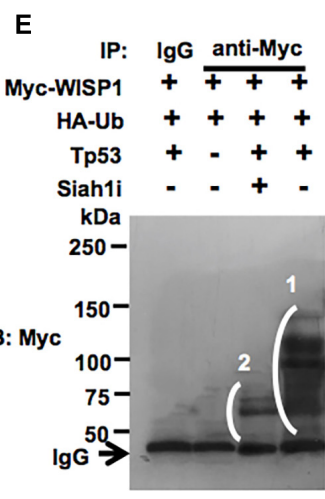
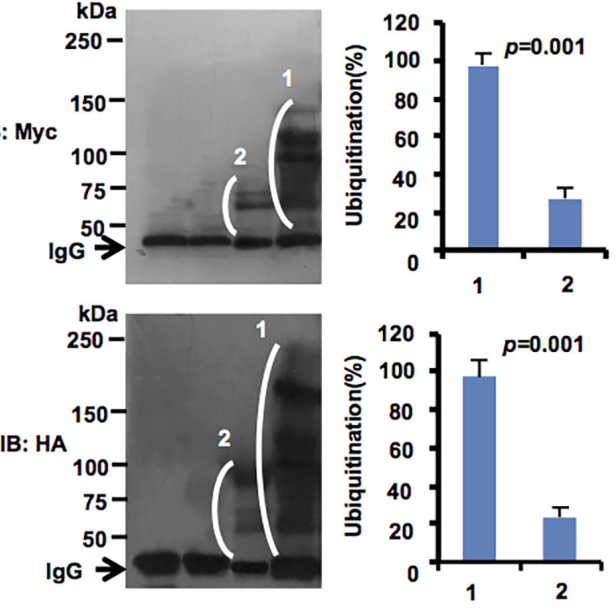

$\mathbf{F}$

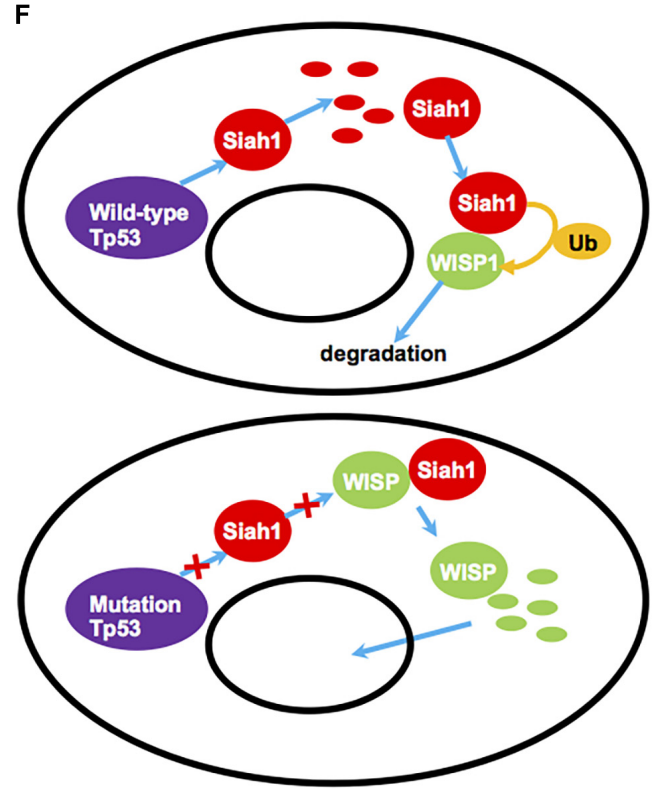

FIGURE 5 | Tp53 mutation may inhibit ubiquitination degradation of Siah1-mediated WISP1. (A) After lentivirus-mediated mouse Tp53 ${ }^{R 172 H}$ overexpression vector or control was stably transduced into SH-PAN cells, lentivirus-mediated human $T p 53^{R 175 H}$ or $T p 53^{R 273 H}$ overexpression vector or control was stably transduced into Capan-2 cells, Western blot analysis was performed to validate Siah1 and WISP1 levels. (B) Capan-2 cells were treated with $10 \mu \mathrm{M}$ of Nutlin-3a at defined time period and were examined by immunoblotting. (C) Panc-1 cells transduced with lentivirus-mediated Siah1 silencing vector or control were treated with $100 \mu \mathrm{g} / \mathrm{ml}$ of cycloheximide (CHX) at defined time periods and were examined for WISP1 expression by Western blot. (D) His6-WISP1 was immobilized on Ni-NTA magnetic agarose beads (columns 2-4) and incubated with GST-Siah-1 (column 4), GST alone (column 3) or without additional protein (column 2). In column 1, GST-Siah-1 was incubated with Ni-NTA magnetic agarose beads without His6-tagged WISP1. After extensive washing, the beads were boiled in SDS sample buffer and the eluted proteins were analyzed by immunoblotting using anti-GST and anti-WISP1 antibodies. In columns 5, 6, and 7, His6-WISP1, GST-Siah-1, and GST alone were run directly as input controls. (E) Myc-tagged WISP1, HA-tagged ubiquitin (Ub), and full-length Tp53 were transiently expressed in Capan-2 cells, as indicated, which were pre-treated with control or Siah-1 shRNA. After treatment with MG132, protein lysates were prepared, immunoprecipitated with anti-Myc antibody or control IgG, and then analyzed by Western blot using anti-Myc antibody (upper) and anti-HA antibody (lower). White brackets indicate smear signals showing poly-ubiquitination. The strong signals at the bottom of the upper image were corresponded to IgG heavy chains. (F) Simplified schematic diagram of the Tp53-Siah1-WISP1 pathway in PDAC cells (wild-type Tp53 or Tp53 mutation). Wild-type Tp53 may promote Siah1 protein levels, which may act as an E3 ubiquitin-protein ligase that mediates ubiquitination and subsequent proteasomal degradation of WISP1. Meanwhile, Tp53 mutation may downregulate Siah1 protein levels, which may inhibit ubiquitination and degradation Siah1-dependent WISP1, and induce WISP1 nuclear import. 
by Western blot at different time points, showed that Siah1 silencing remarkably maintained the protein level of WISP1 (Figure 5C) $(n=3)$. To determine whether Siah1 protein may directly bind to WISP1 protein, a GST-Siah1 fusion protein and a His-tagged WISP1 protein were synthesized by in vitro transcription and translation and used in an immunoprecipitation-immunoblot experiment, Siah-1 was shown to directly interact with WISP1 in vitro (Figure 5D) $(n=3)$. Then, all the data described above motivated us to examine whether Siah-1 is an E3 ubiquitinprotein ligase that mediates ubiquitination and subsequent proteasomal degradation of WISP1. A transient expressionbased assay was performed in which exogenously expressed Tp53, WISP1 (Myc-tagged), and ubiquitin (HA-tagged) induced de novo WISP1 ubiquitination detected by antiMyc antibody or anti-HA antibody in wild-type Tp53 in Capan-2 cells. The inhibition of Siah-1 was accomplished with lentivirus-mediated Siah1 silencing vector which nearly completely abrogated the Tp53-dependent signals detected by anti-Myc antibody (corresponding to the ubiquitination of Myc-tagged WISP1 alone) and by antiHA antibody (Figure 5E) $(n=3)$. Taken together, these data indicate that Siah-1 is essential to Tp53-induced WISP1 ubiquitination.

A simplified schematic diagram of the genetic pathway is illustrated in Figure 5F.

\section{DISCUSSION}

Pancreatic cancer, with an overall 5-year survival rate of $<9 \%$, is the fourth leading cause of cancer death in the past few years, which has the worst prognosis of any major malignancy (Siegel et al., 2018). Compelling data have reported that some major suppressors are involved in pancreatic carcinogenesis, such as p16, Tp53 and Smad4. However, the vehicle that permits early diagnosis or effective treatment for pancreatic cancer is limited. Tp53 is one of the most wellknown pancreatic cancer suppressors that is inactivated in approximately $50-75 \%$ of pancreatic cancers. Alterations of Tp53 protein function permits cells to bypass DNA damage checkpoints and apoptotic signals (Rosenfeldt et al., 2013). Evidence is accumulating that loss of Tp53 function may impose genomic instability in pancreatic cancers ( $\mathrm{Su}$ et al., 2002; Mello et al., 2017). Trp53 $3^{R 172 H}$ mutation cooperates with $\mathrm{Kras}^{G 12 D}$ to promote chromosomal instability and widely metastatic PDAC in mice (Hingorani et al., 2005). Tp53 may have a crosstalk with Wnt signaling. Wild-type Tp53 has been found to inhibit Wnt signaling by different mechanisms including the induction of microRNA-34 (Kim et al., 2011). Loss of Tp53 function increases canonical Wnt signaling through miR-34-specific interactions with target UTRs, whereas miR-34 depletion relieves p53-mediated Wnt repression. Loss of Tp53 or miR-34 contributed to neoplastic progression by triggering the Wnt-dependent, and tissueinvasive activity of colorectal cancer cells. Furthermore, during development, miR-34 interactions with the $\beta$-catenin
UTR determine Xenopus body axis polarity and Wntdependent gene patterning (Sadot et al., 2001; Levina et al., 2004).

To further perceive the downstream molecular alteration in the course of Tp53 mutation in pancreatic carcinogenesis, microarray has been used to explore differentially expressed by genes which may act as potential therapeutic targets. Our previous study has also demonstrated that Tp53 mutation may promote WISP1 expression in mouse PDAC cells (Wang et al., 2015). Recent studies had shown that WISP1 acts as a new oncogene in glioblastoma (Jing et al., 2017), oral squamous cell carcinoma (Jung et al., 2017), gastric cancer (Jia et al., 2017), melanoma (Shao et al., 2016), and colon cancer (Wu et al., 2016). Also, Yang et al. (2015) showed that patients with high expression of WISP-1 had a shorter survival time independent of clinical stage and lymphatic metastasis status in pancreatic cancer. However, the biological function and underlying mechanism of WISP1 in pancreatic carcinogenesis still remains unclear. In our study, we interestingly found that WISP1 protein level was more significantly upregulated in PDAC tissues with Tp53 mutation than in PDAC tissues with wildtype Tp53. And a significant correlation was observed between increased malignant phenotype of tumors from WD-PDAC to $\mathrm{MD}$ - or PD-PDAC and shift from cytoplasmic expression to nuclear accumulation of WISP1. To identify the role of WISP1 in PDAC, its clinical significance and biological function were investigated. Surprisingly, Kaplan-Meier analysis showed that WISP1 expression was probably correlated with the poor prognosis in PDAC patients with only Tp53 mutation. Also, biological behavior analysis showed that WISP1 may promote proliferation, invasion, tumorigenicity, and micrometastasis of mouse PanIN cells and human PDAC cells, which indicated that WISP1 may act as a potential oncogene in pancreatic carcinogenesis. Moreover, Tp53 mutation may promote WISP1 expression in mouse PanIN cells as well as human PDAC cells.

In our study, we showed that Tp53 mutation may contain high expression of WISP1, while "Tp53 mutation" and "WISP1 high expression" presented a close correlation in pancreatic carcinogenesis. Su et al. (2002) showed that WISP1 acts to block cell death at a late-stage in the p53-mediated apoptosis pathway. However, the regulatory mechanism of how Tp53 mutation affects WISP1 expression still remains unclear.

It is well-known that Siah1 encodes a protein that is a member of the seven in absentia homolog (SIAH) family. Besides, the protein is an E3 ligase which is involved in ubiquitination and proteasome-mediated degradation of specific proteins. Ji et al. (2017) showed that Wnt-induced dissociation of the Axin/GSK3 complex permits Siah to interact with Axin which is not associated with GSK3, and promotes Axin degradation mediated by Siah, which represents an important feed-forward mechanism to achieve sustained $W n t / \beta$-catenin signaling. In our study, the data showed that Tp53 mutation inhibited Siah1 expression, which inhibited ubiquitination and degradation of WISP1 mediated by Siah1. 


\section{CONCLUSION}

In summary, our findings show that WISP1 expression was correlated with the poor prognosis in PDAC patients with Tp53 mutation, and WISP1 may act as a potential oncogene in PDAC cells. Tp53 mutation downregulated Siah1 protein levels and further inhibited ubiquitination and degradation of Siah1dependent WISP1 and induced WISP1 nuclear import, which may be a new signaling pathway playing an important role in pancreatic carcinogenesis.

\section{AUTHOR CONTRIBUTIONS}

$\mathrm{WW}, \mathrm{XL}, \mathrm{LmW}$, and TL contributed in data acquisition, data analysis, and interpretation, as well as drafting of manuscript. YZa, YQ, and TB conducted data acquisition, technical support, and statistical analysis. JL and MX conducted cancer casecontrol study. YZh, QW, and LfW contributed in study design, data analysis and interpretation, drafting of manuscript, study's supervision, and critical revision of the manuscript for important intellectual content.

\section{REFERENCES}

Berschneider, B., and Konigshoff, M. (2011). WNT1 inducible signaling pathway protein 1 (WISP1): a novel mediator linking development and disease. Int. J. Biochem. Cell Biol. 43, 306-309. doi: 10.1016/j.biocel.2010.11.013

Chahal, M. S., Ku, H. T., Zhang, Z., Legaspi, C. M., Luo, A., Hopkins, M. M., et al. (2016). Differential expression of Ccn 4 and other genes between metastatic and non-metastatic EL4 mouse lymphoma cells. Cancer Genomics Proteomics 13, 437-442. doi: 10.21873/cgp.20006

Conze, D. B., Wu, C. J., Thomas, J. A., Landstrom, A., and Ashwell, J. D. (2008). Lys63-linked polyubiquitination of IRAK-1 is required for interleukin-1 receptor- and toll-like receptor-mediated NF-kappaB activation. Mol. Cell. Biol. 28, 3538-3547. doi: 10.1128/MCB.02098-07

Deer, E. L., Gonzalez-Hernandez, J., Coursen, J. D., Shea, J. E., Ngatia, J., Scaife, C. L., et al. (2010). Phenotype and genotype of pancreatic cancer cell lines. Pancreas 39, 425-435. doi: 10.1097/MPA.0b013e3181c15963

Fujita, K., Horikawa, I., Mondal, A. M., Jenkins, L. M., Appella, E., Vojtesek, B., et al. (2010). Positive feedback between p53 and TRF2 during telomere-damage signalling and cellular senescence. Nat. Cell Biol. 12, 1205-1212. doi: 10.1038/ ncb 2123

Gurbuz, I., and Chiquet-Ehrismann, R. (2015). CCN4/WISP1 (WNT1 inducible signaling pathway protein 1): a focus on its role in cancer. Int. J. Biochem. Cell Biol. 62, 142-146. doi: 10.1016/j.biocel.2015.03.007

Hingorani, S. R., Petricoin, E. F., Maitra, A., Rajapakse, V., King, C., Jacobetz, M. A., et al. (2003). Preinvasive and invasive ductal pancreatic cancer and its early detection in the mouse. Cancer Cell 4, 437-450. doi: 10.1016/S1535-6108(03) 00309-X

Hingorani, S. R., Wang, L., Multani, A. S., Combs, C., Deramaudt, T. B., Hruban, R. H., et al. (2005). Trp53R172H and KrasG12D cooperate to promote chromosomal instability and widely metastatic pancreatic ductal adenocarcinoma in mice. Cancer Cell 2005, 469-483. doi: 10.1016/j.ccr.2005. 04.023

Hotz, B., Arndt, M., Dullat, S., Bhargava, S., Buhr, H. J., and Hotz, H. G. (2007). Epithelial to mesenchymal transition: expression of the regulators snail, slug, and twist in pancreatic cancer. Clin. Cancer Res. 13, 4769-4776. doi: 10.1158/ 1078-0432.CCR-06-2926

Ji, L., Jiang, B., Jiang, X., Charlat, O., Chen, A., Mickanin, C., et al. (2017). The SIAH E3 ubiquitin ligases promote $\mathrm{Wnt} / \mathrm{beta}$-catenin signaling through mediating Wnt-induced Axin degradation. Genes Dev. 31, 904-915. doi: 10.1101/gad. 300053.117

\section{FUNDING}

This work was supported by National Natural Science Foundation of China (Grant Nos. 81272263, 81672719, and 81702740).

\section{ACKNOWLEDGMENTS}

The authors thank Dr. David Tuveson for providing SH-PAN cell lines.

\section{SUPPLEMENTARY MATERIAL}

The Supplementary Material for this article can be found online at: https://www.frontiersin.org/articles/10.3389/fphar. 2018.00857/full\#supplementary-material

FIGURE S1 | Real-time PCR and Western blot analysis confirmed the efficiency of WISP1 overexpression in SH-PAN cell line and WISP1 knockdown in Panc-1 or MIA PaCa-2 cell line.

Jia, S., Qu, T., Feng, M., Ji, K., Li, Z., Jiang, W., et al. (2017). Association of Wnt1-inducible signaling pathway protein-1 with the proliferation, migration and invasion in gastric cancer cells. Tumour Biol. 39:1010428317699755. doi: $10.1177 / 1010428317699755$

Jing, D., Zhang, Q., Yu, H., Zhao, Y., and Shen, L. (2017). Identification of WISP1 as a novel oncogene in glioblastoma. Int. J. Oncol. 51, 1261-1270. doi: 10.3892/ ijo.2017.4119

Jones, S., Zhang, X., Parsons, D. W., Lin, J. C., Leary, R. J., Angenendt, P., et al. (2008). Core signaling pathways in human pancreatic cancers revealed by global genomic analyses. Science 321, 1801-1806. doi: 10.1126/science.1164368

Jung, E. K., Kim, S. A., Yoon, T. M., Lee, K. H., Kim, H. K., Lee, D. H., et al. (2017). WNT1-inducible signaling pathway protein-1 contributes to tumor progression and treatment failure in oral squamous cell carcinoma. Oncol. Lett. 14, 1719-1724. doi: 10.3892/ol.2017.6313

Kim, N. H., Kim, H. S., Kim, N. G., Lee, I., Choi, H. S., Li, X. Y., et al. (2011). p53 and microRNA-34 are suppressors of canonical. Sci. Signal. 4:ra71. doi: 10.1126/scisignal.2001744

Levina, E., Oren, M., and Ben-Ze'ev, A. (2004). Down-regulation of $\beta$-catenin by p53 involves changes in the rat $\beta$-catenin phosphorylation and Axin dynamics. Oncogene 23, 4444-4453. doi: 10.1038/sj.onc.1207587

Li, J., Kong, D., Wang, Q., Wu, W., Tang, Y., Bai, T., et al. (2017). Niacin ameliorates ulcerative colitis via prostaglandin D2-mediated D prostanoid receptor 1 activation. EMBO Mol. Med. 9, 571-588. doi: 10.15252/emmm.2016 06987

Mello, S. S., Valente, L. J., Raj, N., Seoane, J. A., Flowers, B. M., Mcclendon, J., et al. (2017). A p53 super-tumor suppressor reveals a tumor suppressive p53Ptpn14-Yap Axis in pancreatic cancer. Cancer Cell 32, 460.e6-473.e6. doi: 10. 1016/j.ccell.2017.09.007

Rosenfeldt, M. T., O'prey, J., Morton, J. P., Nixon, C., Mackay, G., Mrowinska, A., et al. (2013). p53 status determines the role of autophagy in pancreatic tumour development. Nature 504, 296-300. doi: 10.1038/nature12865

Sadot, E., Geiger, B., Oren, M., and Ben-Ze'ev, A. (2001). Down-regulation of $\beta$-catenin by activated p53. Mol. Cell. Biol. 21, 6768-6781. doi: 10.1128/MCB. 21.20.6768-6781.2001

Shao, H., Cai, L., Moller, M., Issac, B., Zhang, L., Owyong, M., et al. (2016). Notch1WISP-1 axis determines the regulatory role of mesenchymal stem cell-derived stromal fibroblasts in melanoma metastasis. Oncotarget 7, 79262-79273. doi: 10.18632/oncotarget.13021

Shen, R., Wang, Q., Cheng, S., Liu, T., Jiang, H., Zhu, J., et al. (2013). The biological features of PanIN initiated from oncogenic Kras mutation in genetically 
engineered mouse models. Cancer Lett. 339, 135-143. doi: 10.1016/j.canlet. 2013.07.010

Siegel, R. L., Miller, K. D., and Jemal, A. (2018). Cancer statistics, 2018. CA Cancer J. Clin. 68, 7-30. doi: 10.3322/caac.21442

Sipos, B., Moser, S., Kalthoff, H., Torok, V., Lohr, M., and Kloppel, G. (2003). A comprehensive characterization of pancreatic ductal carcinoma cell lines: towards the establishment of an in vitro research platform. Virchows Arch. 442, 444-452.

Su, F., Overholtzer, M., Besser, D., and Levine, A. J. (2002). WISP-1 attenuates p53mediated apoptosis in response to DNA damage through activation of the Akt kinase. Genes Dev. 16, 46-57. doi: 10.1101/gad.942902

Wang, Q., Jiang, H., Ping, C., Shen, R., Liu, T., Li, J., et al. (2015). Exploring the Wnt pathway-associated LncRNAs and genes involved in pancreatic carcinogenesis driven by Tp53 mutation. Pharm. Res. 32, 793-805. doi: 10.1007/s11095-0131269-z

Wang, Q., Li, J., Wu, W., Shen, R., Jiang, H., Qian, Y., et al. (2016). Smad4dependent suppressor pituitary homeobox 2 promotes PPP2R2A-mediated inhibition of Akt pathway in pancreatic cancer. Oncotarget 7, 11208-11222. doi: 10.18632/oncotarget.7158

Wang, Q., Liu, H., Liu, T., Shu, S., Jiang, H., Cheng, S., et al. (2013). BRCA2 dysfunction promotes malignant transformation of pancreatic intraepithelial neoplasia. Anticancer Agents Med. Chem. 13, 261-269. doi: 10.2174/ 1871520611313020012
Wu, J., Long, Z., Cai, H., Du, C., Liu, X., Yu, S., et al. (2016). High expression of WISP1 in colon cancer is associated with apoptosis, invasion and poor prognosis. Oncotarget 7, 49834-49847. doi: 10.18632/oncotarget. 10486

Yang, J. Y., Yang, M. W., Huo, Y. M., Liu, W., Liu, D. J., Li, J., et al. (2015). High expression of WISP-1 correlates with poor prognosis in pancreatic ductal adenocarcinoma. Am. J. Transl. Res. 7, 1621-1628.

Yuan, F., Chen, X., Liu, J., Feng, W., Wu, X., and Chen, S. Y. (2017). Up-regulation of Siah1 by ethanol triggers apoptosis in neural crest cells through p38 MAPKmediated activation of p53 signaling pathway. Arch. Toxicol. 91, 775-784. doi: 10.1007/s00204-016-1746-3

Conflict of Interest Statement: The authors declare that the research was conducted in the absence of any commercial or financial relationships that could be construed as a potential conflict of interest.

Copyright (C) $2018 \mathrm{Wu}$, Liu, Wei, Li, Zang, Qian, Bai, Li, Xie, Zhu, Wang and Wang. This is an open-access article distributed under the terms of the Creative Commons Attribution License (CC BY). The use, distribution or reproduction in other forums is permitted, provided the original author(s) and the copyright owner(s) are credited and that the original publication in this journal is cited, in accordance with accepted academic practice. No use, distribution or reproduction is permitted which does not comply with these terms. 